\title{
The Galois module of a twisted element in the $p^{m}$-th cyclotomic field
}

\author{
by
}

\author{
KuRT GiRstMair (Innsbruck)
}

1. Introduction and results. For a natural number $n$ let $\xi_{n} \in \mathbb{C}$ denote the primitive $n$th root of unity: $\xi_{n}=e^{2 \pi i / n}$. Then $\mathbb{Q}_{n}=\mathbb{Q}\left[\xi_{n}\right]$ is the $n$th cyclotomic field and $G_{n}=\operatorname{Gal}\left(\mathbb{Q}_{n} / \mathbb{Q}\right)$ is its Galois group over $\mathbb{Q}$. The field $\mathbb{Q}_{n}$ is a module over the group ring $\mathbb{Q}\left[G_{n}\right]$ of $G_{n}$, and, by the normal basis theorem, it is isomorphic to $\mathbb{Q}\left[G_{n}\right]$ itself. In what follows let $n=p^{m}$, where $p$ is a prime number and $m$ a nonnegative integer. Let $q=p^{e}, e \geq 1$, be another power of $p$. For $x \in \mathbb{Q}_{n}$ we consider

$$
x_{n q}=\xi_{n q} x \in \mathbb{Q}_{n q} .
$$

We call $x_{n q}$ the twisted element of $x$, because it arises from $x$ by means of the rotation of the plane $\mathbb{C}$ through $2 \pi /(n q)$. It is almost obvious that the Galois module $\mathbb{Q}\left[G_{n q}\right] x_{n q}$ is contained in $\mathbb{Q}\left[G_{n q}\right] \xi_{n q}$ (cf. proof of Theorem 1 below). Suppose that $d$ is the number of divisors of $p-1$. Then $\mathbb{Q}\left[G_{n q}\right] \xi_{n q}$ is the direct sum of $d$ simple $\mathbb{Q}\left[G_{n q}\right]$-submodules if $p \geq 3$, and it is the direct sum of two simple submodules for $p=2$ and $n q \geq 4$. Hence $\mathbb{Q}\left[G_{n q}\right] \xi_{n q}$ has $2^{d}$ different submodules for $p \geq 3$, and four different submodules for $p=2$, $n q \geq 4$. We consider the case $q \neq 2$ first. Here $\mathbb{Q}\left[G_{n q}\right] x_{n q}$ is always one of the two trivial submodules of $\mathbb{Q}\left[G_{n q}\right] \xi_{n q}$; indeed, we show

Theorem 1. Let $p$ be a prime, $n=p^{m}, q=p^{e}, m \geq 0$, $e \geq 1$. In addition, if $p=2$, let $e \geq 2$. For each element $x \in \mathbb{Q}_{n}, x \neq 0$,

$$
\mathbb{Q}\left[G_{n q}\right] x_{n q}=\mathbb{Q}\left[G_{n q}\right] \xi_{n q} .
$$

For $q=2$ the result is different: Let $M_{1}, M_{2}$ be the simple submodules of $\mathbb{Q}\left[G_{2 n}\right] \xi_{2 n}, n=2^{m} \geq 4$. For $k=1,2$, let

$$
V_{k}=\left\{x \in \mathbb{Q}_{n} ; \mathbb{Q}\left[G_{2 n}\right] x_{2 n}=M_{k}\right\} \cup\{0\} .
$$

Theorem 2. With the above notations, $V_{k}$ is a $\mathbb{Q}$-subspace of $\mathbb{Q}_{n}$ of dimension $\operatorname{dim} V_{k}=n / 4, k=1,2$. Moreover,

$$
\mathbb{Q}_{n}=V_{1} \oplus V_{2} .
$$


2. Proofs. We adopt the above notations. Most of the representation theory of $\mathbb{Q}\left[G_{n}\right]$ we use in the sequel can be found in [1], Section 1, and in [4].

Consider the map

$$
\mathbb{Z} \backslash p \mathbb{Z} \rightarrow G_{n}, \quad k \mapsto \sigma_{k},
$$

where $\sigma_{k}\left(\xi_{n}\right)=\xi_{n}^{k}$. This map is surjective and multiplicative. It is used in order to identify the character group

$$
X_{n}=\left\{\chi: G_{n} \rightarrow \mathbb{C}^{\times} ; \chi \text { a group homomorphism }\right\}
$$

of $G_{n}$ with the group of Dirichlet characters modulo $n$. Indeed, put

$$
\chi(k)= \begin{cases}\chi\left(\sigma_{k}\right) & \text { if } p \nmid k, \\ 0 & \text { otherwise. }\end{cases}
$$

For an element $\alpha=\sum\left\{a_{\sigma} \sigma ; \sigma \in G_{n}\right\}$ in $\mathbb{Q}\left[G_{n}\right]$ let $\chi(\alpha)=\sum a_{\sigma} \chi(\sigma)$ $\in \mathbb{C}$. Let $Y \subseteq X_{n}$ be a conjugacy class of characters, i.e., all characters $\chi$, $\chi^{\prime}$ in $Y$ generate the same group $\langle\chi\rangle=\left\langle\chi^{\prime}\right\rangle$. Then the group ring $\mathbb{Q}\left[G_{n}\right]$ splits into the simple submodules

$$
\mathbb{Q}\left[G_{n}\right]_{Y}=\left\{\alpha \in \mathbb{Q}\left[G_{n}\right] ; \chi(\alpha) \neq 0 \text { only if } \chi \in Y\right\},
$$

of $\mathbb{Q}$-dimension $\operatorname{dim} \mathbb{Q}\left[G_{n}\right]_{Y}=|Y|$.

Next fix $\chi \in X_{n}$. According to [3], [1] there is a map $y(\chi \mid-): \mathbb{Q}_{n} \rightarrow \mathbb{C}$ with the following properties:

(i) $y(\chi \mid-)$ is $\chi$-linear, i.e., for all $\alpha \in \mathbb{Q}\left[G_{n}\right]$ and all $x \in \mathbb{Q}_{n}, y(\chi \mid \alpha x)=$ $\chi(\alpha) y(\chi \mid x)$.

(ii) Let $Y$ be the conjugacy class of $\chi$. Then

$$
\mathbb{Q}_{n, Y}=\left\{x \in \mathbb{Q}_{n} ; y\left(\chi^{\prime} \mid x\right) \neq 0 \text { only if } \chi^{\prime} \in Y\right\}
$$

is the uniquely determined $\mathbb{Q}\left[G_{n}\right]$-submodule of $\mathbb{Q}_{n}$ that is isomorphic to $\mathbb{Q}\left[G_{n}\right]_{Y}$.

The map $y(\chi \mid-)$ is uniquely determined by $\chi$ up to factors in $\mathbb{C}^{\times}$; this means that the maps $c \cdot y(\chi \mid-), c \in \mathbb{C}^{\times}$, are the only ones having properties (i), (ii), too.

Consider the numbers $y\left(\chi \mid \xi_{n}\right) \in \mathbb{C}, \chi \in X_{n}$. Then $y\left(\chi \mid \xi_{n}\right) \neq 0$ iff $\chi$ is a primitive character modulo $n$ (cf. [4]). Hence

$$
\mathbb{Q}\left[G_{n}\right] \xi_{n}=\bigoplus \mathbb{Q}_{n, Y},
$$

where $Y$ runs through the conjugacy classes of primitive characters modulo $n$. We obtain

Lemma 1. Let $n$ be as above, and let $x \in \mathbb{Q}_{n}$. Then

(1) $\mathbb{Q}\left[G_{n}\right] x \subseteq \mathbb{Q}\left[G_{n}\right] \xi_{n}$ iff $y(\chi \mid x)=0$ for all imprimitive characters $\chi \bmod n$; 
(2) $\mathbb{Q}\left[G_{n}\right] x=\mathbb{Q}\left[G_{n}\right] \xi_{n}$ iff $\left\{\chi \in X_{n} ; y(\chi \mid x) \neq 0\right\}=\left\{\chi \in X_{n} ; \chi\right.$ primitive $\}$.

For the proof of Theorem 1 we need two additional lemmas.

Lemma 2. Let $p$ be a prime, $n=p^{m}, q=p^{e}, m \geq 0, e \geq 1$. For $p=2$ let $e \geq 2$.

(1) For each $k \in\{1, \ldots, n\}$ there is a uniquely determined number $j \in$ $\{1, \ldots, n\}$ such that $1+q k \equiv(1+q)^{j} \bmod n q$.

(2) The map $\{1, \ldots, n\} \rightarrow\{1, \ldots, n\}: k \mapsto j$ is bijective.

(3) Let $k, k^{\prime} \in\{1, \ldots, n\}$ and let $j, j^{\prime}$ be their images under the above map. Let $0 \leq l \leq m$. Then $k \equiv k^{\prime} \bmod p^{l}$ iff $j \equiv j^{\prime} \bmod p^{l}$. Furthermore, $k \equiv 0 \bmod p^{l}$ iff $j \equiv 0 \bmod p^{l}$.

The proof of Lemma 2 consists, essentially, in the observation that the subgroups $\{\overline{1+q k} ; k=1, \ldots, n\}$ and $\langle\overline{1+q}\rangle$ of $(\mathbb{Z} / n q \mathbb{Z})^{\times}$coincide and have order $n$ (cf. [2], p. 72 ff.). Note, however, that the map $\mathbb{Z} / n \mathbb{Z} \rightarrow$ $\mathbb{Z} / n \mathbb{Z}: \bar{k} \mapsto \bar{j}$ is not a group homomorphism in general.

Lemma 3. Let $p$ be a prime, $m \geq 2$, and $n=p^{m}$. Let $\beta=\sum\left\{b_{j} \sigma_{j} ; j \in\right.$ $\{1, \ldots, n\}, p \nmid j\} \in \mathbb{Q}\left[G_{n}\right]$ be such that $\beta \xi_{n}=0$. Then $b_{j}=b_{j^{\prime}}$ for all $j, j^{\prime}$ with $j \equiv j^{\prime} \bmod n / p$.

Proof. Put $M=\left\{\alpha \in \mathbb{Q}\left[G_{n}\right] ; \alpha \xi_{n}=0\right\}$. According to Lemma 1, the element $\beta=\sum b_{j} \sigma_{j}$ is in $M$ iff $\chi(\beta)=0$ for each primitive character $\chi \bmod n$. From this we conclude that

$$
\operatorname{dim} M=\mid\left\{\chi \in X_{n} ; \chi \text { is imprimitive }\right\} \mid=\varphi(n / p),
$$

where $\varphi$ is Euler's function. Observe now that $Z^{p}-\xi_{n}^{p}=Z^{p}-\xi_{n / p}$ is the minimal polynomial of $\xi_{n}$ over $\mathbb{Q}_{n / p}$. This means that the trace

$$
T\left(\xi_{n}^{j}\right)=\sum\left\{\sigma_{j^{\prime}} \xi_{n} ; j^{\prime} \equiv j \bmod n / p\right\}
$$

vanishes for each $j, j \in\{1, \ldots, n\}, p \nmid j$. Hence the elements

$$
\alpha_{j}=\sum\left\{\sigma_{j^{\prime}} ; j^{\prime} \in\{1, \ldots, n\}, j^{\prime} \equiv j \bmod n / p\right\},
$$

$j \in\{1, \ldots, n / p\}, p \nmid j$, are in $M$. It is obvious that the $\alpha_{j}$ are $\mathbb{Q}$-linearly independent. So, for dimensional reasons, they form a $\mathbb{Q}$-basis of $M$. When the element $\beta \in M$ is expressed in terms of this basis, the assertion follows.

Proof of Theorem 1. Let $n, q$ be as in Theorem 1. Let

$$
x=\sum_{k=1}^{n} a_{k} \xi_{n}^{k} \in \mathbb{Q}_{n} .
$$

Then $x_{n q}=\sum\left\{a_{k} \xi_{n q}^{1+q k} ; k=1, \ldots, n\right\}$ is a linear combination of primitive $n q$ th roots of unity. Therefore $x_{n q} \in \mathbb{Q}\left[G_{n q}\right] \xi_{n q}$ and $\mathbb{Q}\left[G_{n q}\right] x_{n q} \subseteq \mathbb{Q}\left[G_{n q}\right] \xi_{n q}$. 
Next suppose that $\chi$ is primitive $\bmod n q$. Since $y(\chi \mid-)$ is determined up to factors in $\mathbb{C}^{\times}$only, we may assume that $y\left(\chi \mid \xi_{n q}\right)=1$. Suppose that $y\left(\chi \mid x_{n q}\right)=0$. We show that $x=0$, which proves the theorem, by Lemma 1 . We use induction with respect to the exponent $m$. Let $m=0$, i.e., $n=1$ and $x \in \mathbb{Q}$. Then $0=y\left(\chi \mid x_{q}\right)=y\left(\chi \mid x \xi_{q}\right)=x \cdot y\left(\chi \mid x \xi_{q}\right)=x$.

Now let $m>0$, which means $p \mid n$, and let $n^{\prime}=n / p$. The induction hypothesis is as follows: Let $q^{\prime}=p^{e^{\prime}}, e^{\prime} \geq 1\left(e^{\prime} \geq 2\right.$ for $\left.p=2\right), x^{\prime} \in \mathbb{Q}_{n^{\prime}}$, and $\chi^{\prime}$ a primitive character $\bmod n^{\prime} q^{\prime}$; if $y\left(\chi^{\prime} \mid x_{n^{\prime} q^{\prime}}^{\prime}\right)=0$ then $x^{\prime}=0$.

Take $x$ as above. Then

$$
y\left(\chi \mid x_{n q}\right)=\sum_{k=1}^{n} a_{k} \chi(1+q k)=0 .
$$

For each $j \in\{1, \ldots, n\}$ we put $b_{j}=a_{k}$, where $k$ is the uniquely determined number in $\{1, \ldots, n\}$ with $(1+q)^{j} \equiv 1+q k \bmod n q$ (Lemma 2). Observe that $\eta=\chi(1+q)$ is a primitive $n$th root of unity (use [2], p. 212, and Lemma 2). Now

$$
y\left(\chi \mid x_{n q}\right)=\sum_{j=1}^{n} b_{j} \eta^{j}=0 .
$$

Consider the case $n=p$ first. Because $1+Z+\ldots+Z^{n-1}$ is the minimal polynomial of $\eta$ over $\mathbb{Q}$, all the coefficients $b_{j}$ are equal. Therefore $a_{1}=$ $\ldots=a_{n}$ and $x=0$. Suppose now that $n=p^{m}, m \geq 2$. Put

$$
x^{\prime}=\sum\left\{a_{k} \xi_{n}^{k} ; k \in\{1, \ldots, n\}, p \mid k\right\}
$$

and $x^{\prime \prime}=x-x^{\prime}$. The "trace argument" in the proof of Lemma 3 shows that $T\left(\eta^{j}\right)=0$, for all $j \in\{1, \ldots, n\}, p \nmid j\left(T\right.$ is the trace of $\mathbb{Q}_{n}$ over $\left.\mathbb{Q}_{n^{\prime}}\right)$. Therefore $y\left(\chi \mid x_{n q}^{\prime \prime}\right)=0$, which implies that $y\left(\chi \mid x_{n q}^{\prime}\right)=y\left(\chi \mid x_{n q}\right)-$ $y\left(\chi \mid x_{n q}^{\prime \prime}\right)=0$. However, $x_{n q}^{\prime}$ is the same as $x_{n^{\prime} q^{\prime}}^{\prime}$, with $n^{\prime}=n / p, q^{\prime}=q p$. The induction hypothesis yields $x^{\prime}=0$. Let

$$
\beta=\sum\left\{b_{j} \sigma_{j} ; j \in\{1, \ldots, n\}, p \nmid j\right\} \in \mathbb{Q}\left[G_{n}\right] .
$$

Then $\beta \eta=y\left(\chi \mid x_{n q}^{\prime \prime}\right)=0$. By Lemma 3, the coefficients of $\beta$ fulfill: $b_{j}=b_{j^{\prime}}$ for all $j, j^{\prime} \in\{1, \ldots, n\}, p \nmid j, j^{\prime}, j \equiv j^{\prime} \bmod n^{\prime}$. Then $a_{k}=a_{k^{\prime}}$ for all $k, k^{\prime} \in\{1, \ldots, n\}, p \nmid k, k^{\prime}, k \equiv k^{\prime} \bmod n^{\prime}$. We obtain

$$
x^{\prime \prime}=\sum_{\substack{k=1 \\ p \nmid k}}^{n^{\prime}} a_{k} \sum_{\substack{k^{\prime}=1 \\ k^{\prime} \equiv k \bmod n^{\prime}}}^{n} \xi_{n}^{k^{\prime}}=\sum_{\substack{k=1 \\ p \nmid k}}^{n^{\prime}} a_{k} T\left(\xi_{n}^{k}\right) .
$$

But the traces in the last sum vanish, whence $x^{\prime \prime}=0$ and $x=x^{\prime}+x^{\prime \prime}=0$ follows.

Proof of Theorem 2. Let $n=2^{m}, m \geq 2$. There are exactly two conjugacy classes of primitive characters $\bmod 2 n$, viz. the set of even and 
the set of odd primitive characters (cf. [2], p. 212). Choose an arbitrary odd character $\chi_{1}$ and an arbitrary even character $\chi_{2}$, both of them primitive. Then

$$
\begin{aligned}
& M_{1}=\left\{z \in \mathbb{Q}\left[G_{2 n}\right] \xi_{2 n} ; y\left(\chi_{1} \mid z\right)=0\right\}=\mathbb{Q}\left[G_{2 n}\right]\left(\xi_{2 n}+\xi_{2 n}^{-1}\right), \\
& M_{2}=\left\{z \in \mathbb{Q}\left[G_{2 n}\right] \xi_{2 n} ; y\left(\chi_{2} \mid z\right)=0\right\}=\mathbb{Q}\left[G_{2 n}\right]\left(\xi_{2 n}-\xi_{2 n}^{-1}\right),
\end{aligned}
$$

are the simple submodules of $\mathbb{Q}\left[G_{2 n}\right] \xi_{2 n}$. For each $\sigma \in G_{2 n}, \chi_{k}(\sigma)$ is an $(n / 2)$ th root of unity, $k=1,2$, which shows that the $\mathbb{Q}$-linear map

$$
g_{k}: \mathbb{Q}_{n} \rightarrow \mathbb{Q}_{n / 2}, \quad x \mapsto g_{k}(x)=y\left(\chi_{k} \mid \xi_{2 n} x\right),
$$

is well defined. Let $V_{k}$ denote the kernel of $g_{k}, k=1,2$. Then

$$
V_{k}=\left\{x \in \mathbb{Q}_{n} ; \xi_{2 n} x \in M_{k}\right\}=\left\{x \in \mathbb{Q}_{n} ; \mathbb{Q}\left[G_{2 n}\right] x_{2 n}=M_{k}\right\} \cup\{0\},
$$

since $M_{k}$ is simple. Moreover,

$$
\operatorname{dim} V_{k}=\varphi(n)-\operatorname{dim} g_{k}\left(\mathbb{Q}_{n}\right) \geq \varphi(n)-\varphi(n / 2)=n / 4, \quad k=1,2 .
$$

But $V_{1} \cap V_{2}=\{0\}$, so $\operatorname{dim}\left(V_{1} \oplus V_{2}\right) \geq n / 2=\operatorname{dim} \mathbb{Q}_{n}$. Thus $V_{1} \oplus V_{2}=\mathbb{Q}_{n}$ and $\operatorname{dim} V_{k}=n / 4, k=1,2$.

ExAmPle. Let $n=2^{m}$ and $m \geq 2$ be as above. Consider the elements

$$
x^{+}=1+\xi_{n}^{-1}, \quad x^{-}=1-\xi_{n}^{-1}
$$

in $\mathbb{Q}_{n}$. Then $\xi_{2 n} x^{+}=\xi_{2 n}+\xi_{2 n}^{-1}, \mathbb{Q}\left[G_{2 n}\right] x_{2 n}^{+}=M_{1}, \xi_{2 n} x^{-}=\xi_{2 n}-\xi_{2 n}^{-1}$, $\mathbb{Q}\left[G_{2 n}\right] x_{2 n}^{-}=M_{2}$. Furthermore, $\mathbb{Q}\left[G_{n}\right] x^{+}=\mathbb{Q}\left[G_{n}\right] x^{-}=\mathbb{Q} \oplus \mathbb{Q}\left[G_{n}\right] \xi_{n}$. Hence $V_{1}$ and $V_{2}$ cannot be $\mathbb{Q}\left[G_{n}\right]$-modules. Indeed, if they were, $\mathbb{Q} \oplus$ $\mathbb{Q}\left[G_{n}\right] \xi_{n} \subseteq V_{1} \cap V_{2}$ would follow, which is impossible.

Remark. Clearly the results of this note do not depend on the particular choice $\xi_{n}=e^{2 \pi i / n}$ of a primitive $n$th root of unity. This choice was just made for reasons of convenience, e.g., for the sake of the simple relation $\xi_{n q}^{q}=\xi_{n}$.

\section{References}

[1] K. Girstmair, Character coordinates and annihilators of cyclotomic numbers, Manuscripta Math. 59 (1987), 375-389.

[2] H. Hasse, Vorlesungen über Zahlentheorie, Springer, Berlin 1950.

[3] H. W. Leopoldt, Über die Hauptordnung der ganzen Elemente eines abelschen Zahlkörpers, J. Reine Angew. Math. 201 (1959), 119-149.

[4] G. Lettl, The ring of integers of an abelian number field, ibid. 404 (1990), 162-170.

INSTITUT FÜR MATHEMATIK

UNIVERSITÄT INNSBRUCK

TECHNIKERSTR. 25/7

A-6020 INNSBRUCK, ÖSTERREICH 